\title{
Thromboprophylaxis practice patterns and beliefs among physicians treating patients with abdominopelvic cancers at a Canadian centre
}

\author{
Kristen McAlpine, MD \\ Rodney H. Breau, MD, MSc \\ Marc Carrier, MD \\ Philippe D. Violette, MD \\ Christopher Knee, ND, MSc \\ Ilias Cagiannos, MD \\ Christopher Morash, MD \\ Luke T. Lavallée, MDCM, MSc
}

Accepted Jan. 20, 2020

\author{
Correspondence to: \\ L. Lavallée \\ Ottawa Hospital Research Institute \\ Division of Urology \\ The University of Ottawa, General \\ Campus \\ 501 Smyth Rd \\ PO Box 222 \\ Ottawa ON K1H $8 \mathrm{~L} 6$ \\ lulavallee@toh.ca
}

DOI: $10.1503 /$ cjs.015219
Background: There is inadequate high-quality evidence on thromboprophylaxis for patients undergoing surgery for abdominopelvic cancer. We surveyed physicians who treat patients with abdominopelvic cancer to determine current thromboprophylaxis practice patterns and to determine where research is needed.

Methods: We created an online survey with questions on thromboprophylaxis topics, including type of thromboprophylaxis used, timing of initial thromboprophylaxis dose, use of thromboprophylaxis during chemotherapy, use of extended-duration thromboprophylaxis and areas for future research. The survey questions were reviewed by external content experts to ensure they were appropriate and relevant. Surgeons, thrombosis experts and medical oncologists who manage patients with abdominopelvic cancers at 1 large Canadian academic centre were invited to complete the survey between January and April 2019.

Results: Of the 57 physicians invited, 42 (74\%) completed the survey, including 27 surgeons (response rate 79\%), 9 thrombosis experts (response rate $75 \%$ ) and 6 medical oncologists (response rate 55\%). Most surgeons (22 [82\%]) reported using mechanical thromboprophylaxis, whereas only 1 thrombosis expert $(11 \%)$ recommended mechanical thromboprophylaxis. There was substantial variability in the timing of the initial dose of thromboprophylaxis, with $9 / 10$ urologists $(90 \%)$ and all 7 general surgeons giving the first dose intraoperatively, and three-quarters of thoracic surgeons (3/4 [75\%]), gynecologists (3/4 [75\%]) and thrombosis experts $(7 / 9[78 \%])$ starting thromboprophylaxis after surgery. All medical oncologists believed chemotherapy increases the risk of venous thromboembolism, but $4(67 \%)$ reported that they do not routinely prescribe thromboprophylaxis owing to bleeding concerns. Most respondents (35/38 [92\%]) felt there was a need for more research on thromboprophylaxis and indicated willingness to participate in future clinical trials.

Conclusion: Variability exists in contemporary thromboprophylaxis practice patterns among physicians treating patients with abdominopelvic cancer. Future research is needed to standardize care and improve outcomes for patients.

Contexte : On manque de données de qualité élevée sur la thromboprophylaxie chez les patients traités en chirurgie pour un cancer abdomino-pelvien. Nous avons sondé des médecins traitant ces patients afin de déterminer les tendances actuelles relatives à cette pratique et pour cerner les besoins en recherche.

Méthodes : Nous avons créé un sondage en ligne sur la thromboprophylaxie, comprenant des questions sur le type utilisé, le moment d'administration de la dose initiale, le recours durant la chimiothérapie, l'utilisation prolongée et les domaines de recherche à explorer. Les questions ont été validées par des experts de contenu externes, qui ont veillé à ce qu'elles soient appropriées et pertinentes. Des chirurgiens, des experts en thrombose et des oncologues qui s'occupent de patients atteints de cancers abdomino-pelviens dans un grand centre hospitalier universitaire canadien ont été invités à remplir le sondage entrer janvier et avril 2019.

Résultats : Des 57 médecins sollicités, 42 (74\%) ont répondu au sondage, dont 27 chirurgiens (taux de réponse de 79\%), 9 experts en thrombose (taux de réponse de $75 \%$ ) et 6 oncologues (taux de réponse de $55 \%$ ). La majorité des chirurgiens (22 [82 \%]) recouraient à la thromboprophylaxie mécanique, alors qu'un seul expert en thrombose $(11 \%)$ recommandait cette pratique. Le moment d'administration de la dose initiale variait considérablement: 9 urologues sur 10 (90\%) et chacun des 7 chirurgiens généralistes administraient la première dose durant l'opération, alors que les trois quarts des chirurgiens thoraciques (3/4 [75\%]), des gynécologues $(3 / 4[75 \%])$ et des experts en thrombose $(7 / 9[78 \%])$ commençaient la 
thromboprophylaxie après l'intervention. Tous les oncologues étaient d'avis que la chimiothérapie augmentait le risque de thromboembolie veineuse, mais 4 (67\%) ont indiqué qu'ils ne prescrivaient pas d'emblée de thromboprophylaxie en raison des risques de saignements. La plupart des répondants (35/38 [92\%]) considéraient qu'il faudrait étudier davantage la thromboprophylaxie et ont indiqué leur volonté de participer à d'éventuels essais cliniques.

Conclusion : À l'heure actuelle, les pratiques liées à la thromboprophylaxie varient chez les médecins traitant des patients atteints de cancers abdomino-pelviens. Il faudra mener d'autres études pour normaliser la prestation des soins et améliorer les résultats pour les patients.

V enous thromboembolism (VTE), which includes pulmonary embolism and deep vein thrombosis, is the leading cause of noncancer death among patients who undergo surgery for cancer. ${ }^{1}$ Even when patients survive a VTE, months of pharmacologic therapy are recommended, symptoms can cause disability, and the diagnosis itself is a source of anxiety to the patient. ${ }^{2}$ Venous thromboembolism rates are used as an indicator of perioperative quality of care for surgical patients because VTEs are harmful and may be preventable.

Thromboprophylaxis includes mechanical and pharmacologic interventions to prevent the development of VTEs. Thromboprophylaxis has been studied for decades, yet there remains limited high-quality literature on the effectiveness of mechanical or pharmacologic thromboprophylaxis for patients undergoing abdominopelvic surgery for cancer. ${ }^{3-7}$ Guidelines are available to assist physicians when ordering thromboprophylaxis for their patients around the time of surgery. ${ }^{6-8}$ These guidelines acknowledge the substantial limitations in the literature. Many guideline statements are based on expert opinion, low-quality evidence and data extrapolated from other patient populations. Patients undergoing abdominopelvic cancer surgery frequently have numerous risk factors for VTE and bleeding, including prolonged surgery, exposure to chemotherapy, advanced age and active malignant disease. ${ }^{9}$ Balancing VTE prevention and bleeding complications with thromboprophylaxis poses a clinical challenge.

The objectives of the present study were to describe the current thromboprophylaxis patterns and beliefs of physicians caring for patients with abdominopelvic cancers. We also aimed to identify areas in which physician education was felt to be needed and areas in which VTE research should be focused.

\section{Methods}

We created an online survey to address thromboprophylaxis practice patterns and beliefs of physicians who treat patients with abdominopelvic cancers receiving surgery. To create the survey, we performed a literature review to identify current thromboprophylaxis guidelines, tools to assess VTE risk and recent studies on thromboprophylaxis.
We also identified common and controversial aspects of thromboprophylaxis, and areas where further thromboprophylaxis research study was felt necessary.

The key domains addressed by survey questions included type of mechanical or pharmacologic prophylaxis (or both) used, timing of the initial dose of pharmacologic thromboprophylaxis, duration of thromboprophylaxis, use of thromboprophylaxis during neoadjuvant/adjuvant chemotherapy and methods for assessing VTE risk. The survey questions were reviewed by external content experts to ensure they were appropriate and relevant. The final survey was composed of 31 questions (Appendix 1, available at canjsurg.ca/015219-a1). Several questions had graduated answering in which respondents would be asked the second part of the question only if they had responded affirmatively to first part. For example, "Do you routinely recommend/use mechanical VTE prophylaxis perioperatively?" would be followed with "Which type?" only if the physician had answered "Yes" to the first part of the question. Questions were also included to assess how strongly physicians considered a patient's risk of bleeding when determining whether to provide thromboprophylaxis, areas in which respondents felt further study was necessary and whether respondents would be willing to participate in future clinical trials. Respondents' demographic data were also collected.

\section{Participants}

We identified surgeons and thrombosis experts who treat patients with abdominopelvic cancer at 1 tertiary care cancer centre in Ottawa, Ontario, Canada. We recruited physicians from 1 institution to complete the survey as our aim was to better understand the trends and possible variability in current practice patterns and beliefs among physicians working in the same environment. Physicians were not included from multiple institutions to ensure a high response rate and to avoid confounding. Surgeons were invited to participate if a large proportion of their clinical practice involved patients with abdominopelvic cancers (urologists, general surgeons, gynecologic surgeons, thoracic surgeons). We also invited nonsurgeon thrombosis experts to participate, as they frequently comanage or set policy for perioperative thromboprophylaxis. Physicians 
were emailed the online survey to complete, and implied consent was obtained with completion of the survey. One reminder email was sent 2 weeks after the invitation. The survey was conducted between January and April 2019.

We also explored practice patterns and beliefs of medical oncologists at the same institution who provide neoadjuvant/ adjuvant chemotherapy to patients with abdominopelvic cancer. This included medical oncologists whose practice focused mainly on genitourinary or gastrointestinal cancers. Questions not relevant to the practice of a medical oncologist were removed from the survey (e.g., "When do you most commonly start/recommend starting pharmacologic thromboprophylaxis?").

We used descriptive analyses to analyze the survey results and to identify areas of variability in practice patterns or beliefs between and within physician groups.

Institutional approval was obtained at The Ottawa Hospital for this quality-improvement initiative.

\section{Results}

Of the 57 physicians invited to participate, 42 (74\%) responded, including 27/34 surgeons (12 urologists, 7 general surgeons, 4 thoracic surgeons and 4 gynecologists; response rate 79\%), 9/12 thrombosis experts (response rate $75 \%$ ) and $6 / 11$ medical oncologists (response rate 55\%).

\section{Surgeons and thrombosis experts}

The distribution of experience for responding surgeons was balanced, with $11(41 \%), 11(41 \%)$ and $5(18 \%)$ working less than 10,10-20 and more than 20 years, respectively. The majority of surgeons (22 [82\%]) used mechanical thromboprophylaxis, in most cases (21 [95\%]) sequential compression devices placed at anesthesia induction. Pharmacologic thromboprophylaxis was used by 26 surgeons (96\%). Timing of the initial dose of thromboprophylaxis varied between surgical subspecialties. Most thoracic surgeons (3/4 [75\%]) and gynecologists (3/4 [75\%]) began pharmacologic thromboprophylaxis after surgery (the night of surgery [33\%] or postoperative day $1[67 \%])$, whereas the majority of urologists and general surgeons gave the first dose intraoperatively (9/10 [90\%] and 7/7 [100\%], respectively). The pharmacologic thromboprophylaxis used by $22 / 25$ surgeons (88\%) after surgery was low-molecular-weight heparin; the 3 remaining surgeons (12\%) reported using unfractionated heparin.

The majority of responding thrombosis experts (5 [56\%]) had been in practice for 10-20 years. Postoperative mechanical prophylaxis was recommended by 1 thrombosis expert (11\%). All thrombosis experts recommended the use of pharmacologic prophylaxis postoperatively, and $7(78 \%)$ recommended beginning pharmacologic prophylaxis on the first day after sur- gery. All recommended low-molecular-weight heparin for postoperative thromboprophylaxis.

Assessment of VTE risk before initiation of thromboprophylaxis was endorsed by surgeons and thrombosis experts. The most common patient factors that both surgeons and thrombosis experts considered were patient history of VTE, body mass index and renal function (Fig. 1). Surgical factors were considered by 18 surgeons $(67 \%)$ when deciding on VTE prophylaxis for their patients. Surgical factors that were most commonly considered were the type of operation, the surgical approach and the expected duration of the operation (Fig. 2). A smaller proportion of thrombosis experts (5 [56\%]) considered surgical factors when deciding on VTE prophylaxis.

Few surgeons (2 [7\%]) or thrombosis experts (3 [33\%]) used a risk-assessment tool when deciding on thromboprophylaxis. Eighteen surgeons $(67 \%)$ and 7 thrombosis experts (78\%) used guidelines to assist physicians when deciding on thromboprophylaxis. Surgeons who endorsed using a thromboprophylaxis guideline most commonly used a specialty-specific guideline (10/18 [56\%]), and thrombosis experts most commonly used a thrombosis association guideline (5/7 [71\%]). Both surgeons (16/26 [62\%]) and thrombosis experts (8/9 [89\%]) considered a patient's risk of bleeding when deciding on thromboprophylaxis.

Extended-duration prophylaxis was prescribed by $13 / 25$ surgeons (52\%) in some cases and was recommended by 8 thrombosis experts (89\%). Most surgeons and thrombosis experts who reported prescribing or recommending extended-duration prophylaxis used lowmolecular-weight heparin following hospital discharge (12 [92\%] and 8 [89\%], respectively). Most surgeons who reported prescribing extended-duration prophylaxis $(10 / 13$ [77\%]) prescribed a 28 -day postoperative course. Four thrombosis experts (57\%) who recommended extended-duration prophylaxis recommended a 28 -day postoperative course, and $2(29 \%)$ recommended a 14-day postoperative course.

Twenty-three $(66 \%)$ of 35 surgeons and thrombosis experts reported treating patients who receive chemotherapy before or after surgery. Of the 23, 15 (65\%) believed chemotherapy increases the risk of VTE, yet $20(87 \%)$ reported that they do not prescribe thromboprophylaxis to patients during chemotherapy, the main reason being because they felt patient care was directed by the medical oncologists during chemotherapy (14/20 [70\%]).

\section{Medical oncologists}

Four medical oncologists $(67 \%)$ had been in practice for less than 10 years. All 6 medical oncologists who responded to the survey believed that chemotherapy increased the risk of VTE. None prescribed mechanical or pharmacologic prophylaxis during chemotherapy before or 


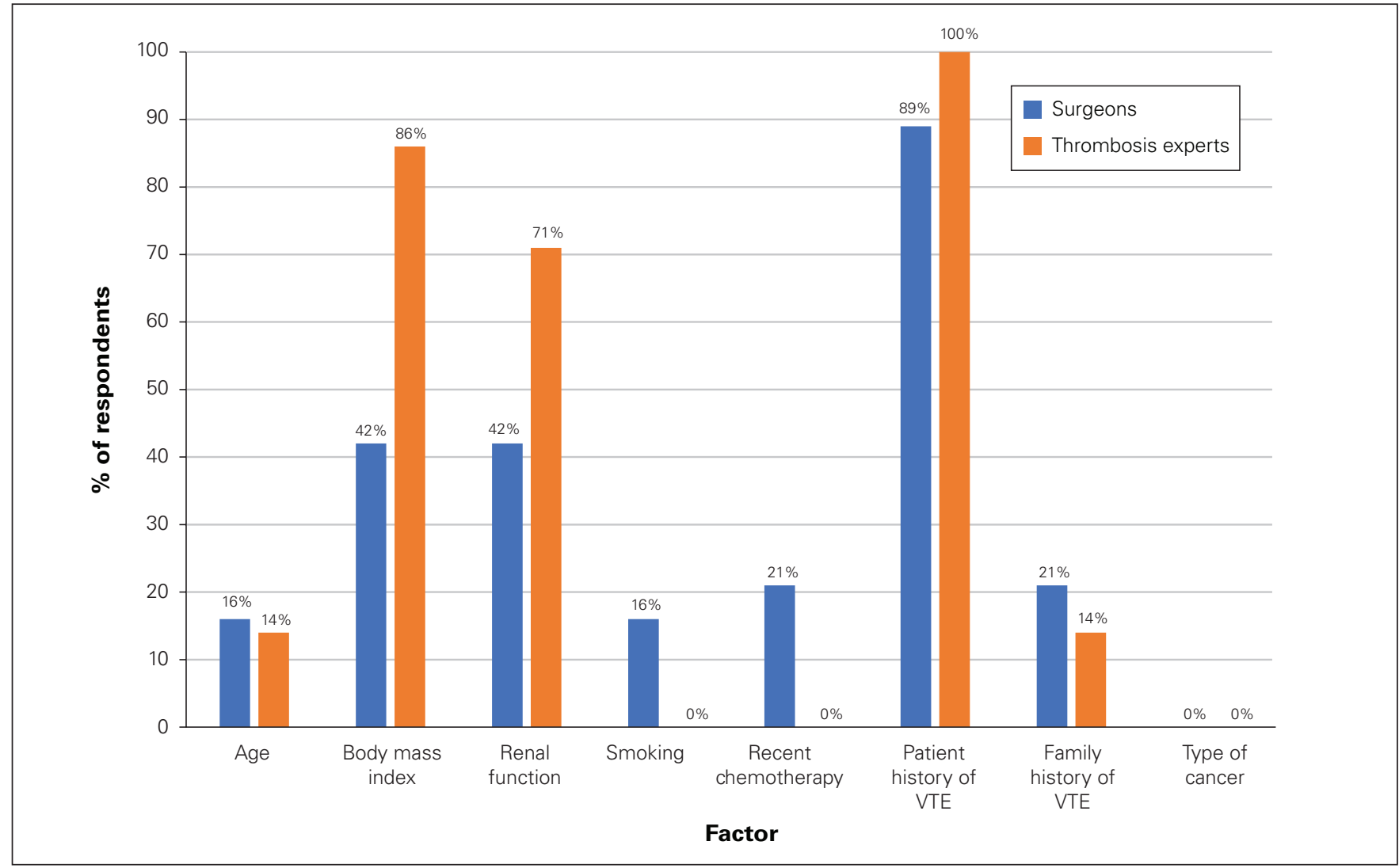

Fig. 1. Patient factors physicians reported considering when choosing thromboprophylaxis. VTE = venous thromboembolism.

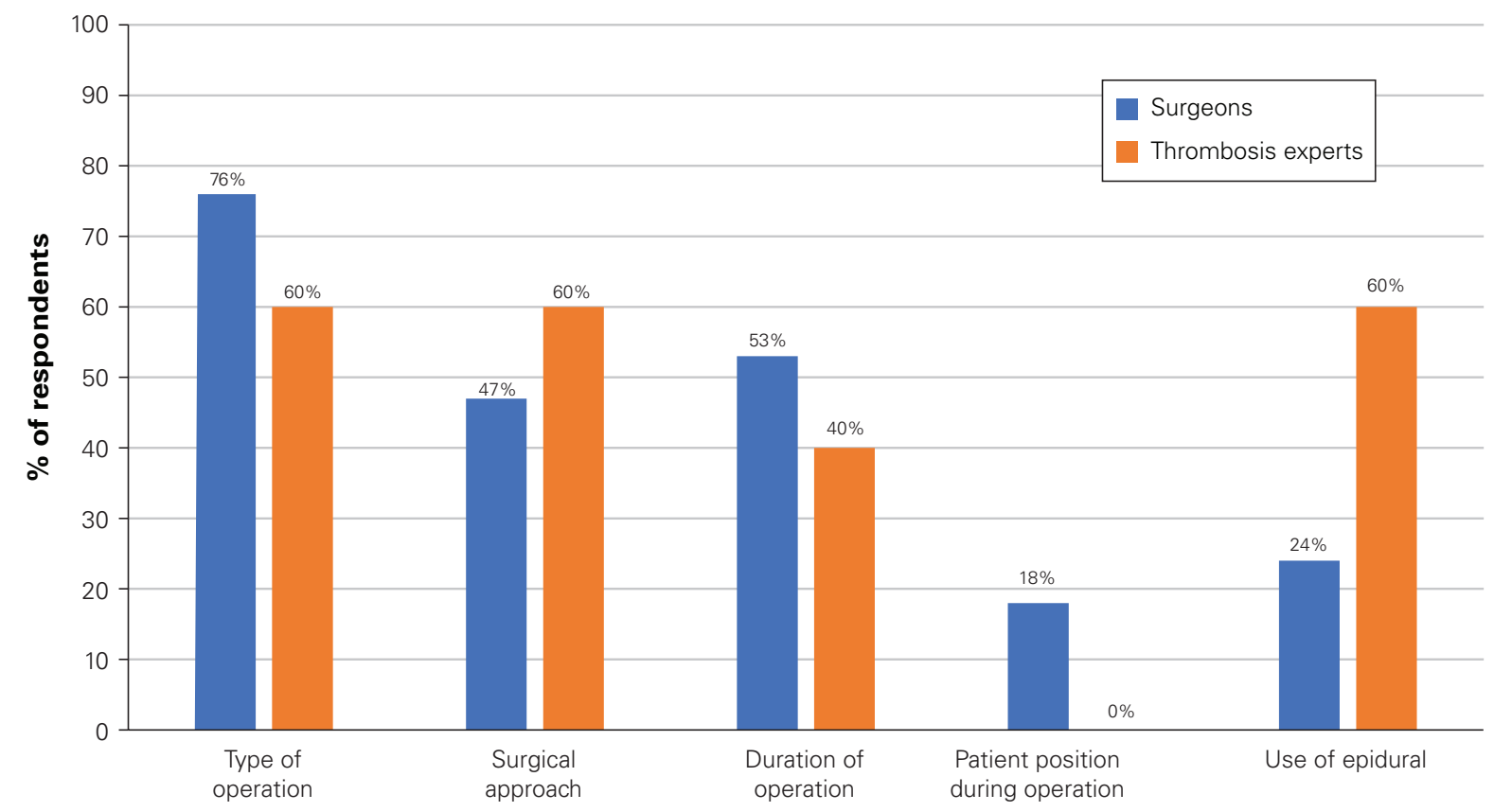

Factor

Fig. 2. Surgical factors physicians reported considering when choosing thromboprophylaxis. 


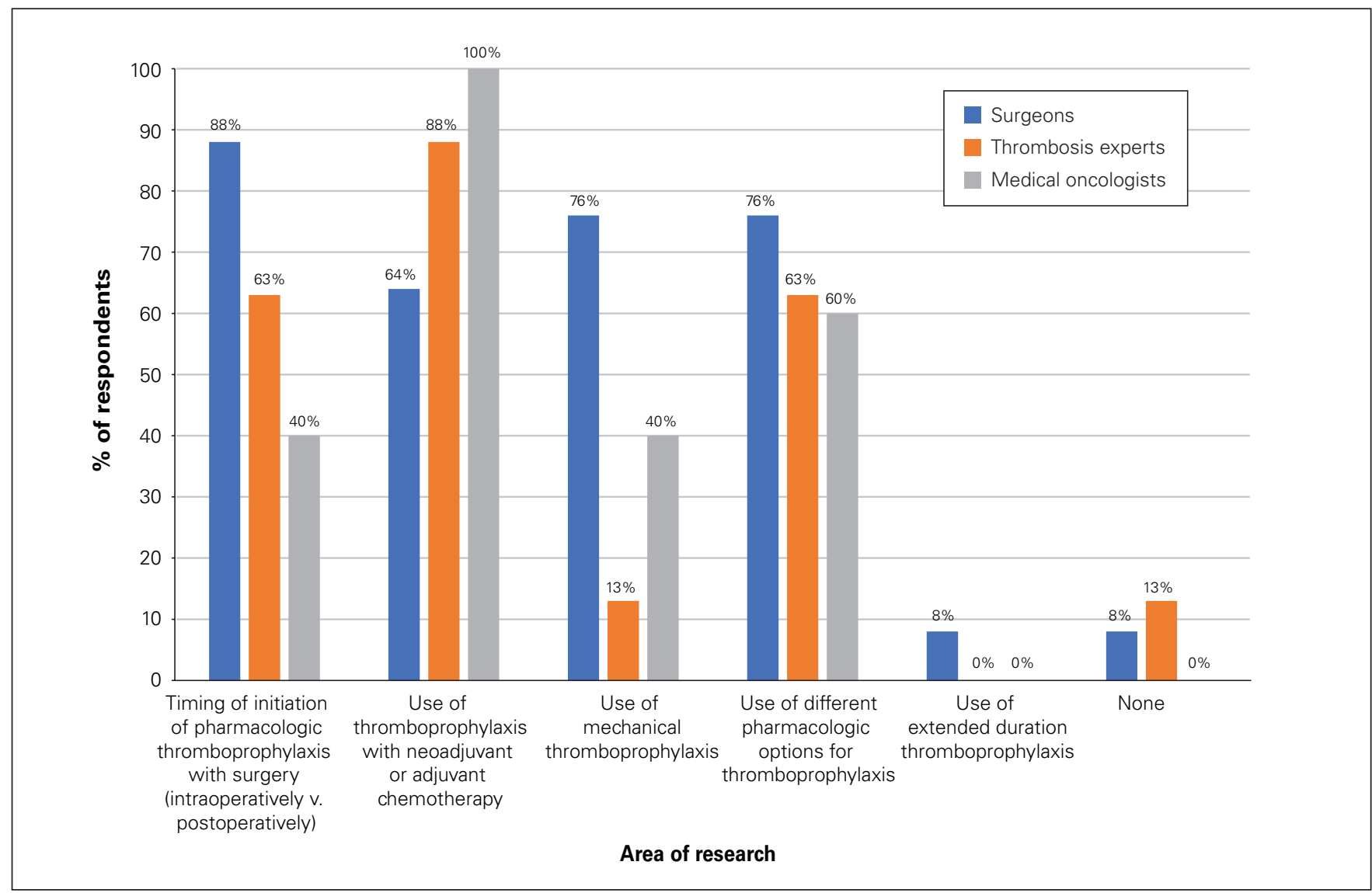

Fig. 3. Physician interest in participating in thromboprophylaxis clinical trials.

after surgery. Medical oncologists did not prescribe pharmacologic prophylaxis during chemotherapy perioperatively because they were concerned about the risk of bleeding $(4[67 \%])$ and because of the cost and inconvenience to patients $(3[50 \%])$.

Two medical oncologists (33\%) considered patient factors when deciding on thromboprophylaxis during chemotherapy. One medical oncologist (17\%) used the Khorana risk assessment score ${ }^{10}$ to assist with thromboprophylaxis ordering, and none used a guideline.

\section{Interest in participating in clinical trials}

There was strong interest among responding physicians in participating in clinical trials investigating perioperative thromboprophylaxis (35/38 [92\%]) (Fig. 3). Surgeons were most interested in a clinical trial investigating the timing of the initial dose of pharmacologic thromboprophylaxis $(22 / 25$ [88\%]). Surgeons also expressed interest in participating in trials on thromboprophylaxis during chemotherapy (16 [64\%]), on mechanical prophylaxis (19 [76\%]) and comparing pharmacologic agents for thromboprophylaxis (19 [76\%]). Thrombosis experts also expressed interest in participating in clinical trials on use of thromboprophylaxis during chemotherapy before or after surgery $(7 / 8[88 \%])$ and on timing of the initial dose of thromboprophylaxis (5 [63\%]). Five of 5 medical oncologists reported interest in participating in a clinical trial investigating thromboprophylaxis use during chemotherapy before or after surgery.

\section{Discussion}

We found substantial variability in the perioperative thromboprophylaxis practice patterns and beliefs among surgeons, thrombosis experts and medical oncologists. Key findings of our study include differences in the timing of the initial dose of thromboprophylaxis perioperatively and in the use of thromboprophylaxis during perioperative chemotherapy. Respondents endorsed the need for clinical trials to further study perioperative thromboprophylaxis and reported interest in participating in these trials.

There is limited clinical overlap in practices of surgeons and thrombosis experts. This may explain why perioperative thromboprophylaxis has been an orphaned research topic for decades, yet advances have been made in several other patient populations for VTE prevention. Multidisciplinary clinical trials that incorporate the knowledge and skill set of both surgeons and thrombosis experts are needed to improve evidence-based and patient-centred care. The majority of our respondents $(92 \%)$ were willing 
to participate in future clinical trials. This buy-in from physicians highlights the understanding that current practice lacks an evidence-based foundation.

We found considerable variability in the practice patterns and beliefs of physicians caring for the same population of patients. For example, the timing of the initial dose of thromboprophylaxis varied drastically by the specialty of the respondent. The majority of urologists and general surgeons reported that they give the initial dose of thromboprophylaxis intraoperatively, whereas most thoracic surgeons, gynecologists and thrombosis experts indicated that they begin pharmacologic thromboprophylaxis postoperatively. Various thromboprophylaxis guidelines are available to assist physicians in determining how to best manage patients' thromboprophylaxis perioperatively. The American College of Chest Physicians guideline on thromboprophylaxis for nonorthopedic surgery has been cited widely and used internationally. ${ }^{8}$ The guideline does not provide explicit guidance as to when thromboprophylaxis should be initiated perioperatively. ${ }^{8}$ Furthermore, more recent subspecialty thromboprophylaxis guidelines give contradictory recommendations regarding when to give the first dose of anticoagulation around the time of cancer surgery. ${ }^{6,7}$ The American Society of Clinical Oncology guideline recommends beginning thromboprophylaxis preoperatively, ${ }^{11}$ whereas the European Urology Association and Canadian Urology Association guidelines recommend giving the initial dose on the day after surgery. ${ }^{6,7}$ These differing recommendations introduce confusion for physicians and highlight the lack of high-quality evidence on the topic. The evidence on the best time to initiate thromboprophylaxis for patients undergoing abdominopelvic surgery for cancer to prevent VTE while avoiding major bleeding is limited. Guideline-based recommendations often rely on studies of patients undergoing orthopedic operations, where thromboprophylaxis timing has been studied more thoroughly. ${ }^{6-8,12}$ In the orthopedic surgery literature, several trials and systematic reviews indicate that the best time to start thromboprophylaxis for orthopedic patients is between 2 hours before surgery and 6-8 hours after surgery. ${ }^{12}$ The results of our survey highlight the need for studies dedicated to answering this question in a patient population undergoing surgery for abdominopelvic cancers. A well-run clinical trial is needed to provide an evidence-based approach to the initial timing of thromboprophylaxis for patients receiving abdominopelvic cancer surgery.

The association between chemotherapy and VTE is well established. ${ }^{913}$ Patients with abdominopelvic cancer are often treated with chemotherapy before or after surgery. Many patients receive platinum-based chemotherapies, which are associated with a higher risk of VTE. ${ }^{13}$ In our study, surgeons and thrombosis experts believed that thromboprophylaxis during chemotherapy could reduce the risk of VTE, but they did not prescribe thromboprophylaxis during chemotherapy as they felt patient care was guided by medical oncologists. No medical oncologists reported prescribing throm- boprophylaxis during neoadjuvant chemotherapy because of perceived bleeding risks and inconvenience to the patient. A recent randomized controlled trial assessing the effect of pharmacologic thromboprophylaxis for patients receiving outpatient chemotherapy for various types of cancer showed that pharmacologic thromboprophylaxis significantly reduced the rate of VTE but also led to a higher rate of major bleeding episodes compared to placebo. ${ }^{14}$ Very few patients were scheduled to receive curative surgery, and very few had localized abdominopelvic cancers. Owing to the results of that study, discussion has been raised regarding whether patients receiving perioperative chemotherapy for abdominopelvic surgery would benefit from pharmacologic thromboprophylaxis. A recent meta-analysis showed that patients who receive neoadjuvant chemotherapy had a $7 \%$ risk of VTE while receiving chemotherapy preoperatively. ${ }^{13}$ Thromboprophylaxis during neoadjuvant chemotherapy has been highlighted as an important area for future research in a recent guideline. ${ }^{6}$ Across all responding physicians in our survey, the role of pharmacologic thromboprophylaxis during chemotherapy before or after surgery was felt to be the area with the greatest need for research.

Because we limited the survey to 1 institution, the patient population and care environment were consistent, and we were able to limit participation bias, as the response rate was $74 \%$.

\section{Limitations}

Limitations of our study include the fact that limiting the survey to physicians at 1 institution may have biased the results toward practice patterns specific to the institution that are not externally generalizable. Our institution is the largest hospital in Canada and has physicians who have trained at a variety of other institutions. However, at other centres internationally, there may be a local preference for a specific thromboprophylaxis agent or initiation of perioperative prophylaxis that differs from the patterns detected on this survey. We believe the finding of variability in perioperative thromboprophylaxis patterns is likely applicable at other centres. Other Canadian surveys on perioperative thromboprophylaxis among surgeons and thrombosis experts have highlighted variability in practices. ${ }^{15-17}$ Our study and others support the lack of evidence-based practice for perioperative thromboprophylaxis across surgical subspecialties in Canada and the need for additional studies to improve this knowledge gap. Second, we developed the questions for the survey after completing a literature review. There may be other components of thromboprophylaxis that were not included in the survey that physicians more strongly feel merit further research. Finally, we did not include community-based physicians in our cohort. This may have affected the survey results; however, we do not believe this would negate the variability in thromboprophylaxis practice patterns or the call for further research. 


\section{Conclusion}

We found substantial variability in the use of thromboprophylaxis among surgeons, thrombosis experts and medical oncologists who care for patients with abdominopelvic cancers. Clinical trials are needed to provide evidence on the risk-to-benefit of thromboprophylaxis for these patients at high risk for VTE.

Affiliations: From the Division of Urology, University of Ottawa, Ottawa, Ont. (McAlpine, Breau, Knee, Cagiannos, Morash, Lavallée); the Ottawa Hospital Research Institute, University of Ottawa, Ottawa, Ont. (Breau, Carrier, Knee, Cagiannos, Morash, Lavallee); the Department of Medicine, University of Ottawa and The Ottawa Hospital, Ottawa, Ont. (Carrier); and the Departments of Health Research Methods, Evidence, and Impact and of Surgery, McMaster University, Hamilton, Ont. (Violette).

Competing interests: Marc Carrier reports grant funding from BristolMyers Squibb, LEO Pharma and Pfizer, and consulting fees from Bayer, Bristol-Myers Squibb, LEO Pharma, Sanofi and Servier, outside the submitted work. Christopher Morash participated in an advisory board run by Sanofi, outside the submitted work. Luke Lavallée reports a quality-improvement grant from Sanofi and advisory board participation for AbbVie, Bayer, Sanofi and Ferring, outside the submitted work. No other competing interests were declared.

Contributors: K. McAlpine, R. Breau, C. Knee, C. Morash and L. Lavallée designed the study. K. McAlpine, C. Knee, I. Cagiannos, C. Morash and L. Lavallée acquired the data, which K. McAlpine, R. Breau, M. Carrier, P. Violette, C. Knee, C. Morash and L. Lavallée analyzed. K. McAlpine, R. Breau and L. Lavallée wrote the article, which all authors critically reviewed. All authors gave final approval of the article to be published.

\section{References}

1. Agnelli G, Bolis G, Capussotti L, et al. A clinical outcome-based prospective study on venous thromboembolism after cancer surgery. Ann Surg 2006;243:89-95.

2. Benelhaj NB, Hutchinson A, Maraveyas A, et al. Cancer patients' experiences of living with venous thromboembolism: a systematic review and qualitative thematic synthesis. Palliat Med 2018;32:1010-20.

3. Harris WH, Salzman EW, Athanasoulis C, et al. Comparison of warfarin, low molecular weight dextran, aspirin, and subcutaneous heparin in prevention of venous thromboembolism following total hip replacement. 7 Bone foint Surg Am 1974;56:1552-62.
4. Kakkar VV, Cohen AT, Edmonson RA, et al. Low molecular weight versus standard heparin for prevention of venous thromboembolism after major abdominal surgery. Lancet 1993;341:259-65.

5. Mismetti P, Laporte S, Darmon JY, et al. Meta-analysis of low molecular weight heparin in the prevention of venous thromboembolism in general surgery. Br 7 Surg 2001;88:913-30.

6. Violette PD, Lavallée LT, Kassouf W, et al. Canadian Urological Association guideline on perioperative thromboprophylaxis and management of anticoagulation. Can Urol Assoc 7 2018;13:105-14.

7. Tikkinen KAO, Cartwright R, Gould MK, et al. EAU guidelines on thromboprophylaxis in urological surgery. 2017. Available: https://uroweb.org/wp-content/uploads/EAU-Pocket-Guidelines -Thromboprophylaxis-2019.pdf (accessed 2019 Aug. 25).

8. Gould MK, Garcia DA, Wren SM, et al. Prevention of VTE in nonorthopedic surgical patients: antithrombotic therapy and prevention of thrombosis, 9th ed: American College of Chest Physicians evidence-based clinical practice guidelines [correction in Chest 2012; 141:1369]. Chest 2012;141(2 Suppl):e227S-77S.

9. Caprini JA. Risk assessment as a guide to thrombosis prophylaxis. Curr Opin Pulm Med 2010;16:448-52.

10. Khorana AA, Kuderer NM, Culakova E, et al. Development and validation of a predictive model for chemotherapy-associated thrombosis. Blood 2008;111:4902-7.

11. Key NS, Khorana AA, Kuderer NM, et al. Venous thromboembolism prophylaxis and treatment in patients with cancer: ASCO Clinical Practice Guideline update. 7 Clin Oncol 2019:38:496-520.

12. Hull RD, Pineo GF, Stein PD, et al. Timing of initial administration of low-molecular-weight heparin prophylaxis against deep vein thrombosis in patients following elective hip arthroplasty: a systematic review. Arch Intern Med 2001;161:1952-60.

13. Di Nisio M, Candeloro M, Rutjes AWS, et al. Venous thromboembolism in cancer patients receiving neoadjuvant chemotherapy: a systematic review and meta-analysis. 7 Thromb Haemost 2018;16: 1336-46.

14. Carrier M, Abou-Nassar K, Mallick R, et al. Apixaban to prevent venous thromboembolism in patients with cancer. $N$ Engl 7 Med 2019;380:711-9.

15. Agzarian J, Linkins LA, Schneider L, et al. Practice patterns in venous thromboembolism (VTE) prophylaxis in thoracic surgery: a comprehensive Canadian Delphi survey. 7 Thorac Dis 2017;9:80-7.

16. Hopkins L, Carrier M, Plante M, et al. Surgical venous thromboprophylaxis: a cross-sectional survey of Canadian gynaecologic oncologists. 7 Obstet Gynaecol Can 2012;34:673-7.

17. Al Rawahi B, Le Gal G, Auer R, et al. A survey of thrombosis experts evaluating practices and opinions regarding venous thromboprophylaxis in patients post major abdominal surgery. Thromb f 2017;15:2. 